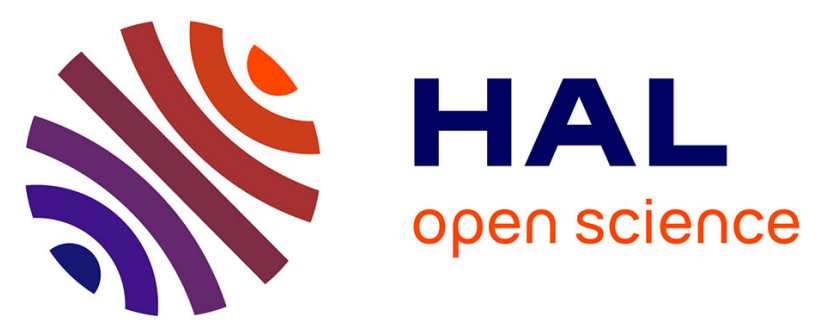

\title{
Root compression on MRI compared to clinical findings in patients with recent onset cervical radiculopathy
}

Barbara Kuijper, Jos Th.J. Tans, Bas F van Der Kallen, Frans Nollet, Geert J Lycklama a Nijeholt, Marianne de Visser

\section{- To cite this version:}

Barbara Kuijper, Jos Th.J. Tans, Bas F van Der Kallen, Frans Nollet, Geert J Lycklama a Nijeholt, et al.. Root compression on MRI compared to clinical findings in patients with recent onset cervical radiculopathy. Journal of Neurology, Neurosurgery and Psychiatry, 2010, 82 (5), pp.561. 10.1136/jnnp.2010.217182 . hal-00589970

\section{HAL Id: hal-00589970 https://hal.science/hal-00589970}

Submitted on 3 May 2011

HAL is a multi-disciplinary open access archive for the deposit and dissemination of scientific research documents, whether they are published or not. The documents may come from teaching and research institutions in France or abroad, or from public or private research centers.
L'archive ouverte pluridisciplinaire HAL, est destinée au dépôt et à la diffusion de documents scientifiques de niveau recherche, publiés ou non, émanant des établissements d'enseignement et de recherche français ou étrangers, des laboratoires publics ou privés. 


\section{Root compression on MRI compared to clinical findings in patients with recent onset}

cervical radiculopathy

Barbara Kuijper *†

Jos Th.J.Tans *

Bas F. van der Kallen (radiologist 1)I

Frans Nollet $\ddagger$

Geert J. Lycklama a Nijeholt (radiologist 2)d

Marianne de Visser $\S$

Medical Centre Haaglanden

* Department of Neurology

II Department of Radiology

P.O Box 432

2501 CK The Hague, The Netherlands

$\dagger$ Maasstad Hospital

Department of Neurology

P.O.Box 9119

3078 HT Rotterdam, The Netherlands

Academic Medical Centre

$\ddagger$ Department of Rehabilitation

$\S$ Department of Neurology

P.O. Box 22660

1100 DD Amsterdam, The Netherlands

Corresponding author: Barbara Kuijper

Maasstad Hospital

Department of Neurology

P.O.Box 9119

3078 HT Rotterdam, The Netherlands

phone 0031102911911, fax 0031102911072

kuijperb@maasstadziekenhuis.nl 


\section{Abstract \\ Objectives}

To evaluate occurrence of symptomatic and asymptomatic root compression caused by herniated discs and spondylotic foraminal stenosis by magnetic resonance imaging (MRI) in patients with recent onset cervical radiculopathy.

\section{Participants}

78 patients with symptoms and signs of cervical radiculopathy of less than one month's duration.

\section{Methods}

We determined the clinically suspected level of root compression in each patient. Two neuroradiologists independently evaluated MRI's, blinded for the clinical findings. For each patient the level of root compression on MRI was compared with the clinically affected level. We also examined the cause of compression: herniated disc, spondylotic foraminal stenosis or both.

\section{Results}

In 73 percent of patients the clinically affected root was compressed on MRI. In 45 percent MRI showed root compression without clinical substrate together with, or to a lesser extent without, the coexistence of compression of the clinically affected root. MRI's were assessed as normal in 13-15 percent of cases and in 9-10 percent only asymptomatic roots were compressed.

Herniated discs without spondylosis were more often responsible for root compressions only at the clinically affected level and spondylotic foraminal stenosis for multiple root compression including compression of clinically unaffected roots.

\section{Conclusion}

MRI findings in patients with cervical radiculopathy should be interpreted together with the clinical findings as false positive and false negative MRI's occur rather frequently.

\section{Keywords}

Cervical radiculopathy, Magnetic resonance imaging (MRI), disc herniation, spondylotic foraminal stenosis 
Licence for Publication:

The Corresponding Author has the right to grant on behalf of all authors and does grant on behalf of all authors, an exclusive licence (or non exclusive for government employees) on a worldwide basis to the BMJ Publishing Group Ltd to permit this article (if accepted) to be published in JNNP and any other BMJPGL products and sublicenses such use and exploit all subsidiary rights, as set out in our licence. (http://group.bmj.com/products/journals/instructionsfor-authors/licence-forms)

Competing Interest: None declared. 


\section{Introduction}

Root compression without clinical substrate as a coincidental finding on MRI of the cervical spine is well known. ${ }^{1-4}$ However, presence of a clinically evident cervical radiculopathy without root compression on MRI does also occur. ${ }^{5-7}$ Knowledge of the occurrence of this phenomenon is important to take correct treatment decisions.

The most common causes of cervical root compression are narrowing of the foraminal space secondary to spondylarthrosis and herniated discs. ${ }^{8-10}$ Large studies investigating the relationship between the radiological and clinical findings are lacking.

In this study we assess the relation between the clinically affected level of root compression and the level of root compression on magnetic resonance imaging (MRI) in a group of patients with recent onset cervical radiculopathy.

\section{Methods}

\section{$\underline{\text { Subjects }}$}

In our prospective cohort study we included patients with a clinical diagnosis of unilateral cervical radiculopathy in whom efficacy of either a cervical collar or physiotherapy was compared with a wait and see policy. ${ }^{11}$ The diagnosis cervical radiculopathy was made by a neurologist according to the following inclusion criteria: radiation of arm pain distal to the elbow, symptoms for less than one month, arm pain on a visual analogue scale of $40 \mathrm{~mm}$ or more, plus at least one of the following: (1) worsening of the arm pain by neck movements, (2) sensory symptoms in one or more adjacent dermatomes, (3) diminished deep tendon reflexes in the affected arm, or (4) muscle weakness in one or more adjacent myotomes. The clinically affected level was determined by the neurologist via structured history taking and physical examination data.

Written informed consent was obtained from all patients. The medical ethics committees of the participating hospitals approved the protocol.

\section{$\underline{\text { MRI protocol }}$}

MRI was performed at 1.5 Tesla, and included sagittal proton density (PD) and T2 weighted turbo spin-echo imaging (TSE; TR/TE: 2900/23/182; echo train length (ETL): 5) and T1 weighted TSE (TR/TE 664/13; ETL: 3), using $3 \mathrm{~mm}$ thick slices and pixels of $1 \mathrm{~mm}^{2}$. Axial imaging consisted of a T2 weighted MEDIC sequence (TR/TE 1140/27) and T1 TSE (TR/TE 538/13, ETL: 3) using $3 \mathrm{~mm}$ thick slices and $1 \mathrm{~mm}^{2}$ pixels. Axial slices were placed perpendicular to the vertebral bodies and were angulated in the same way. Axial imaging 
covered intervertebral spaces C4 to T1. Per patient four levels were investigated on both sides: C4-5 (root C5), C5-6 (root C6), C6-7 (root C7) and C7-Th1 (root C8).

\section{MRI evaluation protocol}

The MRI examinations were evaluated independently by two neuroradiologists who were blinded to clinical findings. For each cervical level, presence of herniated disc and bony foraminal stenosis by spondylarthrosis was assessed.

The probability of root compression, either caused by herniated disc or spondylotic foraminal stenosis, was scored on a five-point scale: 'definitely no root compression', 'possibly no root compression', 'indeterminate', 'possibly root compression' and 'definitely root compression'. Presence of other abnormalities like spinal canal stenosis with or without cord compression, tumors, and other findings was recorded but not included in the analysis.

\section{$\underline{\text { Analysis }}$}

Data on root compression were dichotomized as either root compression (possibly and definitely root compression) or no root compression (definitely and possibly no root compression and indeterminate).

For each patient we compared the level of root compression with that of the clinically affected root. As it is not always feasible to determine with certainty the level of root compression by history and physical examination, ${ }^{12}$ we separately recorded the presence of root compression one level higher or lower. We also examined the cause of compression: herniated disc, spondylotic foraminal stenosis or both.

\section{Results}

From May 2005 to December 200682 patients with a clinical diagnosis of recent onset unilateral cervical radiculopathy underwent MRI of the cervical spine.

Four scans were of poor quality because of movement artifacts by pain $(n=2)$ and claustrophobia ( $\mathrm{n}=2) .78$ MRI's were fully examined.

Data on the clinical signs of radiculopathy in these 78 patients are listed in table 1 . Sensory abnormalities were found in $89.9 \%$ of the patients, diminished reflexes in $48.1 \%$ and muscle weakness in $29.1 \%$. Two patients $(2.6 \%)$ only experienced pain. The mean age was 47.4 year (range 24-70). There was no difference in age between patients with herniated discs and spondylotic foraminal stenosis. 
Table 1

Clinical signs and symptoms in 78 patients with suspected cervical radiculopathy

\begin{tabular}{|l|l|}
\hline & $\begin{array}{l}78 \text { patients of total } \\
\text { group } \\
\text { No }(\%)\end{array}$ \\
\hline only pain & $2(2.6)$ \\
\hline muscle weakness & $23(29.1)$ \\
\hline diminished reflexes & $38(48.1)$ \\
\hline sensory abnormalities & $70(89.9)$ \\
\hline
\end{tabular}

In table 2 single and multiple root compression assessments are listed. The clinically affected root was judged as compressed in 73.1/69.2\% (radiologist 1/2) of all cases. When we consider compression of the roots adjacent to the clinically affected level as symptomatic root compression, this percentage increased to $78.2 / 74.4 \%$.

Single level, one sided root compression was found in $47.4 \%$ of patients. Most of these compressions were of the clinically affected root, according to table 2 in $35.9 / 34.6 \%$ (radiologist 1/2). Multiple root compression was seen in 39.7/37.2\% of cases (radiologist 1/2), mostly a combination of clinically affected and unaffected roots. One or more clinically unaffected root compressions were reported in 44.9/46.2\% of all patients. MRI's were assessed as normal in $12.8 / 15.4 \%$ of cases and in $9.0 / 10.3 \%$ only unaffected roots were compressed. We additionally recorded the clinical signs listed in table 1 , for the group of patients on whom the 2 neuroradiologists agreed that the MRI showed no root compression at the clinical level or one level above or below. No difference was found between the clinical data of patients with and without root compression on MRI. 
Table 2

Single and multiple root compression assessed on MRI in 78 patients with suspected cervical radiculopathy

\begin{tabular}{|c|c|c|}
\hline total $n=78$ & radiologist 1 & radiologist 2 \\
\hline Single root compression on MRI & $\mathrm{n}=37(47.4)$ & $\mathrm{n}=37(47.4)$ \\
\hline Compression of clinically affected root only & $28(35.9)$ & $27(34.6)$ \\
\hline $\begin{array}{l}\text { Compression of the root one level higher or lower than } \\
\text { that of the clinically affected root }\end{array}$ & $3(3.8)$ & $2(2.6)$ \\
\hline Compression of the root two levels higher or lower & $1(1.3)$ & $1(1.3)$ \\
\hline Compression of contralateral root & $5(6.4)$ & $7(9.0)$ \\
\hline Multiple root compression on MRI & $\mathrm{n}=31(39.7)$ & $\mathrm{n}=29(37.2)$ \\
\hline $\begin{array}{l}\text { Compression of clinically affected root together with } \\
\text { compression one level higher or lower }\end{array}$ & $2(2.6)$ & $1(1.3)$ \\
\hline $\begin{array}{l}\text { Compression of clinically affected root and } \\
\text { contralateral root compression } \\
\text { Compression of clinical affected root together with } \\
\text { one level higher or lower and contralateral root } \\
\text { compression } \\
\text { Compression of root one level higher and lower } \\
\text { together with contralateral root compression }\end{array}$ & $18(23.1)$ & $13(16.7)$ \\
\hline Compression of multiple contralateral roots & $1(1.3)$ & 0 \\
\hline Normal MRI & $10(12.8)$ & $12(15.4)$ \\
\hline
\end{tabular}

Herniated discs were the single cause of root compression in $41.1 / 34.6 \%$ and spondylotic foraminal stenosis in 35.9/21.7\% of our patients (table 3). A combination of herniated disc and spondylotic foraminal stenosis was reported in 10.3/28.2\%. Herniated discs were mainly seen in the group with one compressed root, whereas spondylotic foraminal stenosis more often caused multiple root compressions, including compression of clinically unaffected roots (see table 3). 
Table 3

Causes of root compression in 78 patient with suspected cervical radiculopathy who underwent MRI

\begin{tabular}{|l|l|l|}
\hline total $\mathrm{n}=78$ & radiologist 1 & radiologist 2 \\
\hline single root compression & $\mathrm{n}=37(47.4)$ & $\mathrm{n}=37(47.4)$ \\
\hline -by herniated disc & $25(32.1)$ & $24(30.8)$ \\
\hline -by spondylotic foraminal stenosis & $10(12.8)$ & $4(5.1)$ \\
\hline -by both & $2(2.6)$ & $9(11.5)$ \\
\hline \hline multiple root compression & $\mathrm{n}=31(39.7)$ & $\mathrm{n}=29(37.2)$ \\
\hline -by herniated disc(s) & & \\
\hline -by spondylotic foraminal stenosis & $18(23.1)$ & $3(3.8)$ \\
\hline -by both & $6(7.7)$ & $13(16.7)$ \\
\hline \hline Normal MRI & $10(12.8)$ & $13(16.7)$ \\
\hline
\end{tabular}




\section{Discussion}

To the best of our knowledge this is the first study evaluating the correlation between clinical signs and symptoms and the occurrence of root compression on MRI in a well defined population of patients with recent onset unilateral cervical radiculopathy, in a setting closely resembling clinical practice.

In almost three quarter of the 78 patients the clinical level corresponded with the level of root compression on MRI. Assessment of the level of root compression by neurological examination alone is reported to be difficult. ${ }^{13-18}$ Inclusion of the root compressions one level above or below the clinically suspected level, led to an increase of the percentage of matching clinical and MRI levels by only 5 percent for both radiologists indicating that the localising value of the neurological examination is quite good.

Strikingly, we found false positive root compressions in 45 percent of the cases, defined as compressions on the contralateral, i.e., asymptomatic, side or at least two levels higher or lower than the clinically affected level. These asymptomatic root compressions were often present together with root compression at the clinically affected level or adjacent to this. In order to avoid unnecessary treatment of root compressions observed on MRI without clinical substrate, physicians should only interpret MRI results after a careful history and neurological examination.

Earlier studies reported a high percentage of cervical degenerative disease on MRI in asymptomatic patients, with a higher prevalence in older age. Teresi et al studied 100 MRI's of the cervical spine of patients who were investigated for laryngeal disease. Twenty percent of patients aged 45-54 years and 57 percent of patients older than 64 years had cervical disc protrusion without clinical symptoms. ${ }^{19}$ Another study on asymptomatic subjects showed 'major' abnormalities (herniated disc, foraminal stenosis, disc space narrowing) in 28 percent

of people over 40 years of age. ${ }^{4}$ However, these studies only investigated asymptomatic patients, whereas our study included patients based on the presence of cervical radiculopathy at one level.

We also found a fair amount of false negative MRI's. MRI's were assessed as normal in 12.8 /15.4\% of cases and in 9.0/10.3\% only asymptomatic roots were compressed. So, together we had 21.8/25.6 percent of false negative MRI's.

Patients were eligible for our study if they fulfilled strict inclusion criteria. We feel confident that they had indeed cervical radiculopathy because all patients except two had neurological deficits corresponding with radicular pain, and no other conditions emerged during a 6- 
months follow-up period. We had low percentages of surgery so we could not confirm our diagnosis surgically in most cases. ${ }^{11}$

The cause of the root compression was most often herniated disc, particularly in those patients with unilateral MRI abnormalities only at the clinically affected level. This is noteworthy since it is often assumed that spondylotic foraminal stenosis is the most common cause of root compression in cervical radiculopathy. ${ }^{8-10} 12$ Our study shows that foraminal stenosis was more frequently asymptomatic. The occurrence of herniated discs and spondylotic changes was not related to age.

We previously found a high interobserver agreement of $91 \%$ for MRI evaluation of root compression with a kappa score of 0.67 . This agreement was less for the cause of the compression, i.e., herniated disc (81\%) and spondylotic foramen stenosis (82\%) (unpublished data, manuscript accepted for publication in Clinical Radiology). In the present study the two neuroradiologists also disagreed more on assessment of herniated discs and foraminal stenoses than on the presence of root compression. Particularly the reported percentages of spondylotic foraminal stenosis differed (table 3). It is known that on MRI spondylotic foraminal stenosis is often more difficult to detect and CT-myelography techniques are probably more accurate. ${ }^{5-7} 20$ Our neuroradiologists may have underreported spondylotic foraminal stenosis, although the 55 and 63 percent of patients in whom stenosis was found by radiologist 1 and 2 suggest otherwise (unpublished data, manuscript accepted for publication in Clinical Radiology).

Another limitation is that the neuroradiologists knew that all patients in the study had a clinical diagnosis of cervical radiculopathy. They may have been more aware of the possibility of root compression in general, resulting in higher percentages abnormal MRI's. Because the radiologists had no information on the level and side of the radiculopathy the main results of our study on the relation between clinical and MRI findings seem reliable.

\section{Conclusion and clinical implications}

It is evident from this study that MRI findings in patients with cervical radiculopathy are only meaningful in a clinical context. False negative MRI results were encountered in almost one quarter, and false positive results in half of the patients. Therefore, cervical MRI is only useful when there is a clear picture of cervical radiculopathy. 
1. Jensen MV, Tuchsen F, Orhede E. Prolapsed cervical intervertebral disc in male professional drivers in Denmark, 1981-1990. A longitudinal study of hospitalizations. Spine 1996;21:2352-5.

2. van Rijn JC, Klemetso N, Reitsma JB, et al. Observer variation in the evaluation of lumbar herniated discs and root compression: spiral CT compared with MRI. Br J Radiol 2006;79:372-7.

3. van Rijn JC, Klemetso N, Reitsma JB, et al. Observer variation in MRI evaluation of patients suspected of lumbar disk herniation. AJR Am J Roentgenol 2005;184:299303.

4. Boden SD, Davis DO, Dina TS, et al. Abnormal magnetic-resonance scans of the lumbar spine in asymptomatic subjects. A prospective investigation. J Bone Joint Surg Am 1990;72:403-8.

5. Fortin J, Riethmiller DW, Vilensky JA. No clear winner in differing imaging modalities for cervical radiculopathy. Pain Physician 2002;5:285-7.

6. Bartlett RJ, Hill CR, Gardiner E. A comparison of T2 and gadolinium enhanced MRI with CT myelography in cervical radiculopathy. Br J Radiol 1998;71:11-9.

7. Birchall D, Connelly D, Walker L, et al. Evaluation of magnetic resonance myelography in the investigation of cervical spondylotic radiculopathy. Br J Radiol 2003;76:525-31.

8. Tanaka N, Fujimoto Y, An HS, et al. The anatomic relation among the nerve roots, intervertebral foramina, and intervertebral discs of the cervical spine. Spine 2000;25:286-91.

9. Adams C. Cervical spondylotic radiculopathy and myelopathy. Amsterdam/ New York / Oxford: Elsevier Publishing Company, 1976.

10. Radhakrishnan K, Litchy WJ, O'Fallon WM, et al. Epidemiology of cervical radiculopathy. A population-based study from Rochester, Minnesota, 1976 through 1990. Brain 1994;117:325-35.

11. Kuijper B, Tans JT, Beelen A, et al. Cervical collar or physiotherapy versus wait and see policy for recent onset cervical radiculopathy: randomised trial. Bmj 2009;339:b3883.

12. Kuijper B, Tans JT, Schimsheimer RJ, et al. Degenerative cervical radiculopathy: diagnosis and conservative treatment. A review. Eur J Neurol 2009;16:15-20.

13. Fisher MA. Electrophysiology of radiculopathies. Clin Neurophysiol 2002;113:317-35.

14. Levine MJ, Albert TJ, Smith MD. Cervical Radiculopathy: Diagnosis and Nonoperative Management. J Am Acad Orthop Surg 1996;4:305-316.

15. Yoss RE, Corbin KB, Maccarty CS, et al. Significance of symptoms and signs in localization of involved root in cervical disk protrusion. Neurology 1957;7:673-83.

16. Ozgur BM, Marshall LF. Atypical presentation of C-7 radiculopathy. J Neurosurg 2003;99:169-71.

17. Slipman CW, Plastaras C, Patel R, et al. Provocative cervical discography symptom mapping. Spine J 2005;5:381-8.

18. Narayan P, Haid RW. Treatment of degenerative cervical disc disease. Neurol Clin 2001;19:217-29.

19. Teresi LM, Lufkin RB, Reicher MA, et al. Asymptomatic degenerative disk disease and spondylosis of the cervical spine: MR imaging. Radiology 1987;164:83-8.

20. Kaiser JA, Holland BA. Imaging of the cervical spine. Spine 1998;23:2701-12. 